\title{
The Syntax in Pantomimic Re-enactments of Events Among Polish Participants
}

\begin{abstract}
What appears to be a key question in recent studies on language evolution is the notion of natural word order - a hypothesis propounding word order to be innate in a phylogenetic and cognitive sense (Dryer, 2005; Pagel, 2009; Gell-Mann \& Ruhlen, 2011). Gesture and sign studies provide a sound base for the topic. The primary idea for the study comes from Goldin-Meadow et al. (2008) research, which proposed the silent gesture paradigm, in which participants communicate simple events with the aid of their hands. The result of their research suggests that when participants communicate via gestures, notwithstanding their native language, they apply the SOV (subject - object - verb) word order - a finding which has been largely substantiated, but also discussed by following studies (e.g., Gibson et al., 2013; Hall et al., 2013). The purpose of our study was to verify whether the SOV order is prevalent in the experimental environment when participants are instructed to use whole-bodily pantomime rather than hand-and-arm gesture only. The actors in the study were instructed to perform reversible events from the pictures. Reversible events means that both the actor and the patient can perform an action. There exists an exciting opportunity for our study to shed a new light on research into natural word order.
\end{abstract}

\section{Keywords:}

pantomime, natural word order, transitive events

1 Nicolaus Copernicus University in Toruń, Centre for Language Evolution Studies, Poland, E-MAIL: monika.feba@vp.pl.

2 Nicolaus Copernicus University in Toruń, Centre for Language Evolution Studies, Poland. 


\section{INTRODUCTION}

An important issue in recent language evolution studies is the so-called "natural word order", i.e., a word order that may be primary in a cognitive and phylogenetic sense (Dryer, 2005; Pagel, 2009; Gell-Mann \& Ruhlen, 2011). Important insights into this topic come from gesture and sign language studies. Following GoldinMeadow et al. (2008), researchers have used the 'silent gesture' paradigm, in which participants communicate simple events using their hands. Goldin-Meadow et al. (2008) established that when communicating in the manual-bodily channel, regardless of the word order in their native language, participants tend to rely on the SOV order (subject - object - verb) - a finding that has been largely corroborated, but also qualified in some ways, by other studies (e.g., Gibson et al., 2013; Hall et al., 2013; Sandler et al., 2005). In our study, we aimed at checking whether the SOV order still prevails when participants communicate with whole-body pantomime rather than purely manual gesture.

We used existing recordings of pantomimic representations of simple transitive events (Wacewicz et al., under review). This material consisted of 80 short (5-20 seconds) video recordings of 5 Polish student mimetically enacting simple events, such as "a girl slaps a woman" or "a man waves to a girl”, based on a matrix of cartoon-like pictures. Importantly, the actors represented the events by whole-body pantomime rather than manual gesture. The events were reversible, meaning that both the agent and the patient could perform the action (Hall et al., 2013). We analysed those re-enactments to establish their "syntactic" structure: two ELAN-trained coders annotated the recordings for the segments representing the subject (the agent), the verb (the action) and the object (the patient) separately, so as to determine the order in which these were performed.

\section{LITERATURE OVERVIEW}

The present section of our paper presents the literature regarding the subject of our study. It provides a theoretical introduction as well as discusses previous research in the topic.

\section{A HANDFUL OF DEFINITIONS}

In the present paper we describe various linguistic phenomena with the use of certain metalanguage. To disambiguate, we state our metalanguage here. First of all, we use the words syntax and word order to refer to the order of constituents 
within a sentence, and not in the Chomskyan sense of the study of principles and processes which govern the means by which sentences are constructed (Chomsky, 1957). All of the examples which we analyse in our experiment include transitive events, which are sentences involving a subject and an object. We also refer to these entities as the agent and the patient. The agent, which in English is usually the subject of the sentence, refers to an entity which performs the action (Crystal, 2008). The patient, on the other hand, is the entity affected by the action which the verb denotes (Crystal, 2008). The transitive events which constitute the stimulus and are re-enacted by participants are reversible and non-reversible. The reversible events are these constructions where both the agent and the patient are animate. This means that both of them can perform the action denoted by the verb; as a result, the sentence's interpretation is subject to ambiguity. The type of events in which only the agent is animate are called non-reversible events (Gibson et al., 2013).

\section{PANTOMIME}

Pantomime stems from mimesis which serves re-enactment and representation of events and can be used in communicative contexts incorporating a range of vocal, facial and bodily modalities (Donald, 1991, pp. 169-170, 177). It is crucial that mimesis is purposeful and children use it to communicate during pre-linguistic stages (1991, p. 171). Hence, pantomime can be defined as a theatrical or artistic expression of sequence-like narration patterns (McNeill, 1992, p. 37). It is not characterized by conventionality and systematicity (Zlatev, 2007, p. 320). McNeill defines pantomime as a "dumb-show [of gestures], a gesture or sequence of gestures conveying a narrative line, with a story to tell, produced without speech" (Pre-print for The Encyclopaedia of Language and Linguistics, Gesture: A Psycholinguistic Approach, p. 3, doa: 18.02.2016), where body actions depict objects and events which are further reconstructed and re-enacted in a communicative way.

\section{NATURAL WORD ORDER}

Every language in the world has a word order; however, languages vary in how stable their word order is (Gershkoff-Stowe \& Goldin-Meadow, 2002). Languages like English are on the one side of the spectrum, having a strict word order; those like Walpiri land on the other side, being less rigid and allowing for all logical word orders (Gershkoff-Stowe \& Goldin-Meadow, 2002). Gershkoff-Stowe \& Goldin-Meadow (2002) hypothesize that there may be an underlying, pre-defined order of conceptualising events. 


\section{THE NATURAL WORD ORDER - IS IT REALLY SO DIFFERENT FOR SPEECH AND GESTURAL REPRESENTATION?}

Goldin-Meadow et al. (2008) propose that gestural re-construction of language gives insight into natural word order (NWO) - the native syntax of human communication. In their study, they attempt to check whether a speaker's native language influences the syntactic structure of their non-verbal narration. The idea is based primarily on children's language acquisition which is a proof that syntactic pattern is acquired in during the early stages of language development. Therefore, in the research, Goldin-Meadow et al. use a twofold approach based on events selected from children's narrations: (a) a representation of a communicative task in which the participants use gestures to describe an event, and (b) a representation of a non-communicative task in which a participant reconstructs the events with pictures. Furthermore, to intensify the linguistic variety, the participants come from four various backgrounds, as the group includes 10 English speakers, 10 Chinese speakers, 10 Turkish speakers and 10 Spanish speakers.

English, Chinese and Spanish speakers use the SVO word order in their speech, presenting the (1) actor who performs the (2) action done to (3) some animate or inanimate object. This pattern, however, in not common for the speakers of Turkish, where the syntax reflects the SOV structure - starting with the (1) actor and moving to the (2) animate or inanimate object to or with whom the (3) action is done. The authors argue that if the language is rooted deeply into our representations, the order of events in non-verbal reconstructions of actions should reflect the speech pattern - it would be mapped onto various modalities.

The participants were given two tasks:

(1) The gesture task: describing 36 motion events chosen from children's talks. The events were displayed on a computer screen and described by the forty above-mentioned participants in two conditions: verbal description first (to assess the syntax of the speakers); and gestural description second (to assess whether verbal and non-verbal representations match). The events were important as they included such actions as waving, moving, twisting an object, etc., so speakers could adapt to various syntactic patterns to each event.

(2) The transparency task: describing 36 motion events chosen from children's talks. The events were displayed on a computer screen and the participants were given sets of transparent pictures from which they were supposed to construct the events anew placing one transparency on the other. The pictures contained lines such as the direction of twisting a knob, or some movement, and objects that took part in events. This task was conducted in order to verify whether the participants would incorporate the same pattern for the represen- 
tation of an event or whether they would extend it - the order was assessed based on the order of transparencies in a pile.

Speech of each of the participants was analysed in order to assess their syntactic choice for each event presented and to mark semantic elements (actor (Ar), action (A), patient/instrument $(\mathrm{P})$ ). The study revealed that speakers of all languages consistently chose the ArAP structure conforming with the SVO pattern for intransitive actions in place and entailing movement. However, for transitive events the choices split - English and Spanish speakers used the ArAP - SVO patterns, in accordance with their verbal choices; Turkish speakers used ArPA - SOV structure; and Chinese speakers incorporated ArAP - SVO structure for in-place events but ArPA - SOV construction for events involving movement. Next, the results for gestural reconstructions revealed the following: (1) intransitive events in gestural description followed the verbal pattern of narration - ArAP in each language; (2) transitive events caused the speaker to make various choices in their gestural representations - speakers used single gesture orders unlike the pattern of their spoken language. Furthermore, ArPA - SOV was the leading syntactic and semantic pattern which was "natural” for crossing-space actions (actions in which the speaker indicates a position change) in Chinese and both types of actions in Turkish, but not conforming with the syntactic patterns for both types of actions in English and Spanish as well as in in-space actions in Chinese. As for the non-communicative transparency task, the analysis revealed that all speakers maintained the predominant ArPA - SOV pattern while selecting the transparencies, and hence they did not conform to the pattern of their spoken languages but to their gestural representation. Surprisingly, when they were asked to describe the event from the transparency they followed their verbal pattern and not the gestural one.

In conclusion, the authors state that it is striking that participants were consistent with their choices and the major syntactic, and hence semantic, choices were the same for groups across languages in gestural and pictorial reconstructions of events. Furthermore, the pattern of gesture did not conform with the speech pattern of their native language. Based on that, Goldin-Meadow et al. propose that ArPA - SOV pattern may reflect a natural word order for nonverbal representation of events.

\section{A COMMUNICATIVE ACCOUNT OF ORDERING OF EVENTS}

Gibson et al. (2013) argue that the subject precedes the object in a majority of languages due to the fact that in recounting events, people prefer to embody the agent rather than the patient. Furthermore, they indicate that another default feature of languages is positioning the verb in the final position. To substantiate the claim, they mention the Nicaraguan Sign Language, a language which emerged independently from the influence of other linguistic systems. Gibson et al. also refer to 
the experiments conducted by Goldin-Meadow discussed in the previous subsection of the paper. However, this statement contrasts with the fact that a plethora of languages exhibits the SVO word order. In response, they propose that the SVO syntax emerges due to communicative and memory pressure.

These communicative pressures emerge due to the fact that language operates in the noisy channel (Shannon \& Weaver, 1949). The noisy channel hypothesis states that whenever a sender wants to communicate some meaning to a recipient with an utterance, the message is always obscured, which results in an imperfect utterance. The receiver has to use this imperfect utterance in order to decipher the intended meaning. In effect, the sender has to apply a strategy which minimises the noise and facilitates the process of meaning reconstruction for the recipient. The noisy channel hypothesis is the driving force behind and the explanation of the SOV and SVO preference over other syntaxes.

Gibson et al. propose to evaluate the noisy channel hypothesis in the following conditions: when recovering the intended meaning is not problematic due to the semantics of a sentence, in the cases where the agent is animate and the patient is not - called non-reversible events; when recovering the intended meaning may be problematic, in the cases where both the agent and the patient are animate - called reversible events. Although the noisy channel hypothesis operates in a communicative environment, it also applies when an individual encodes a message.

The experiments involved participants first verbally describing and then gesturing events which were presented to them in the form of a mute film. The events included a varying number of participants (from one to three) as well as transitive and intransitive events, both reversible and non-reversible. The experiment was conducted over three groups of language users, namely, of English (SVO), Japanese (SOV), and Korean (SOV).

For the non-reversible events, the English gestured patient before the action, however, for the reversible - the patient before the object. The noisy channel hypothesis seems to account for these preferences. Since both Japanese and Korean are SOV languages, the setup of the experiment required some adaptation to reduce the noise from the participants' mother tongues. The experiments were conducted in two conditions: the first one the same as for the English, and the second one, adapted. The participants of the experiment received stimuli which included the events from the films in the form of a thought bubble or an utterance bubble. The Japanese and the Korean participants gestured mainly (over 90\%) the SOV order for both the reversible and non-reversible events in the first condition. In the second condition, the main verb of the higher-level was gestured in the position right after the subject. With regard to the "reported" sentence, the preference 
for the patient appearing before the action significantly dropped. For the reversible events, the patient was gestured before the action only in $66 \%$ of the cases for the Japanese speakers and 57\% for the Korean speakers, compared to 85\% and 86\% for non-reversible events respectively.

To sum up, Gibson et al. replicated the finding reported in Goldin-Meadow et al. regarding the SOV preference for non-reversible events. However, for the cases where ambiguity may arise due to increasing complexity of a sentence, this preference is dropped.

\section{UNDERSTANDING THE SEQUENCE OF EVENTS THROUGH COGNITIVE CONSTRAINTS}

The next replication of the study initiated by Goldin-Meadow was conducted by Hall et al. (2013) and it is a response to the discrepancies between the results obtained by Gibson et al. (2013) and Meir et al. (2010). Meir et al. found that in case of confusability, the participants switched to the OSV order. However, Hall et al. pinpoint cognitive constraints (role conflict), rather than noise in the channel, as a preference of one syntactic representation over another.

Hall et al. replicated the study in a similar fashion to Gibson et al., only changing the arrangement of the items under the three conditions: (1) non-reversible and reversible events were mixed; (2) first non-reversible, then reversible events; (3) the order of reversible and non-reversible events varied with every participant - reversible first, non-reversible first or mixed. The stimulus was in the form of a video and the participants of the experiment were native English speakers, their main task was to display the events via pantomime. What is more, they were also required to verbally describe what was happening on the videos and also perform one filler exercise.

Unsurprisingly, the verbal description of transitive events from the videos was carried out in the SVO order. In condition (1), the participants pantomimed the SOV (50.9\%) and SVO (27.5\%) orders for non-reversible events and the SVO (35.4\%) and SOV (12.5\%) for reversible events. The SOSV and SOSVO syntaxes were also present. Importantly, some of the participants performed case marking in their pantomime in order to show which role they assumed at a given time, by, for example, showing " 1 ” and " 2 " to refer to the agent and the patient respectively. Other than that, the experiment under the condition (1) replicated earlier findings.

In the randomised condition (2), the non-reversible events were acted out mainly in the SOV manner. However, for the reversible events, the number of SOV re-enactments significantly dropped, to $2.6 \%$. The leading syntax was SVO; yet, other word orders had a greater representations, for instance, OSV, SOSV, SOSVO. This result is important due to the fact that these re-enactments contradict what 
the noisy channel hypothesis predicted, as it had expected the avoidance of S and $\mathrm{O}$ appearing on a single side of V. Finally, some case-marking was also present.

The purpose of condition (3) was to test whether the pantomimic re-enactment of reversible events has any influence on the re-enactment of the non-reversible ones. Some groups of actors received reversible events first, then non-reversible ones, other received the stimulus in the opposite order. The experiment resulted in the conclusions that starting with reversible events had no systematic influence on the SOV order of non-reversible events. However, case-marking was more prevalent in the case when reversible events were presented first, for both reversible and non-reversible events. Other than that, the experiment under condition (3) replicated the findings of the experiments in the previous conditions.

The results of the experiments contradict the noisy channel hypothesis, since strings as OSV, SOSVO and SOSV for reversible events appear and the number of their occurrences increases. According to the noisy channel, these orders should be avoided since they are subject to ambiguous interpretation. As a result, Hall et al. develop a role conflict hypothesis which does not fail in the same way as the noisy channel hypothesis does. The hypothesis assumes that participants always take the roles of animate agents and patients in a sentence; however, the presence of the verb (action) requires from them to assume the agentive role. In this interpretation, only the sequences with $\mathrm{O}$ directly followed by $\mathrm{V}$ may be problematic to understand.

Yet another finding of the experiments was proto-case-marking. Participants, instead of referring to some extra-linguistic referent, devised a system to indicate the role they were assuming. What is more, proto-case-marking was more common for reversible than non-reversible events. Another strategy for avoiding role conflict was using space to indicate a role.

\section{COMPARISON}

The table 1 is a juxtaposition of key notions presented in the previous sections.

\section{Table 1. A comparison of approaches}

\begin{tabular}{|c|c|c|}
\hline Goldin-Meadow et al. & Gibson et al. & Hall et al. \\
\hline $\begin{array}{l}\text { - SOV-preference, independ- } \\
\text { ent of spoken language } \\
\text { pattern for non-reversible } \\
\text { events; } \\
\text { - SOV is believed to predomi- } \\
\text { nate in early stages of spo- } \\
\text { ken and signed languages. }\end{array}$ & $\begin{array}{l}\text { - noisy channel - preference } \\
\text { of SVO order over SOV; } \\
\text { - case-marking; } \\
\text { - communicative and memory } \\
\text { pressures influence the word } \\
\text { order. }\end{array}$ & $\begin{array}{l}\text { - role conflict; } \\
\text { - case-marking; } \\
\text { - cognition favours some } \\
\text { constituent orders over oth- } \\
\text { ers in the case of reversible } \\
\text { events - against SOV. }\end{array}$ \\
\hline
\end{tabular}




\section{THE RESEARCH}

The present section is devoted to the presentation of our empirical research. First, we give reasons for conducting a replication of the abovementioned studies. Next, we illustrate the material we used in our study. Finally, we describe the analytical process.

\section{NWO AMONG POLISH PARTICIPANTS - THE FIRST STEP}

While searching for a theory on natural word order, we did not come across any studies researching this phenomenon in our native language. Hence, we decided that it may be an exciting opportunity to conduct a similar research to the ones presented above and check how the syntactic and semantic sequence for events and actions resemble Polish and whether speech and gestural patterns vary. To conduct the analyses we used the already-existing database of short clips where actors re-enact simple transitive events.

\section{MATERIAL}

The original material was gathered in the academic year 2014/2015 for the purpose of the project entitled: "Multimodality in Event Re-enactments" presented by Jordan Zlatev (Centre for Languages and Literature, Lund University, Sweden) and Przemysław Żywiczyński and Sławomir Wacewicz (Centre for Language Evolution Studies (CLES), NCU, Toruń, Poland) at Protolang4, 2015, in Rome. Five actors (three female - two male) from The Spinning Globe Students' Theatrical Association, students of Nicolaus Copernicus University, participated in the project. The material amounted to 80 videos $(n=80)-16$ short hand-drawn reversible events re-enacted by 5 actors. All the actors were Polish native speakers who were acting out the events in a communicative way so that the observer could guess the event presented. The pictures contained both positive and negative transitive events (slap, push, kiss, wave) performed by female and male characters, both adults and children. The actors performed particular features such as position or height in their re-enactments to make the characters and events easier to guess.

\section{ANALYSIS}

The videos were annotated and the syntactic patterns marked by two expert judges independently. One annotated and assessed the structures, and the other one eval- 
uated the precision of the analysis. Data were segregated for each actor and the patterns added up.

\section{RESULTS}

The results of our experiments replicate the previous findings for the native speakers of Polish. The SVO syntax constitutes the main pattern in the majority of re-enactments for reversible events $-46 \%$. Still, $27.5 \%$ of instances constituted clause-final action (SOV and OSV) re-enactments. Others involved such representations as $\mathrm{OV}, \mathrm{SV}$, and $\mathrm{O}$. These results were excluded from the analysis as they do not fulfil the requirement of transitivity. Moreover, in a communicative situation, $\mathrm{O}$ can be interpreted as $\mathrm{S}$, and $\mathrm{S}$ as $\mathrm{O}$, which is yet another argument for their disqualification.

\section{DISCUSSION}

The present paper is the result of our initial investigation into the natural word order and pantomimic re-enactment of transitive events. Our results confirm the hypothesis of role-conflict proposed by Hall et al. (2013) since the SVO word order was found in the majority of re-enactments performed by native speakers of Polish. However, we also attested verb-final word orders, with OSV being more

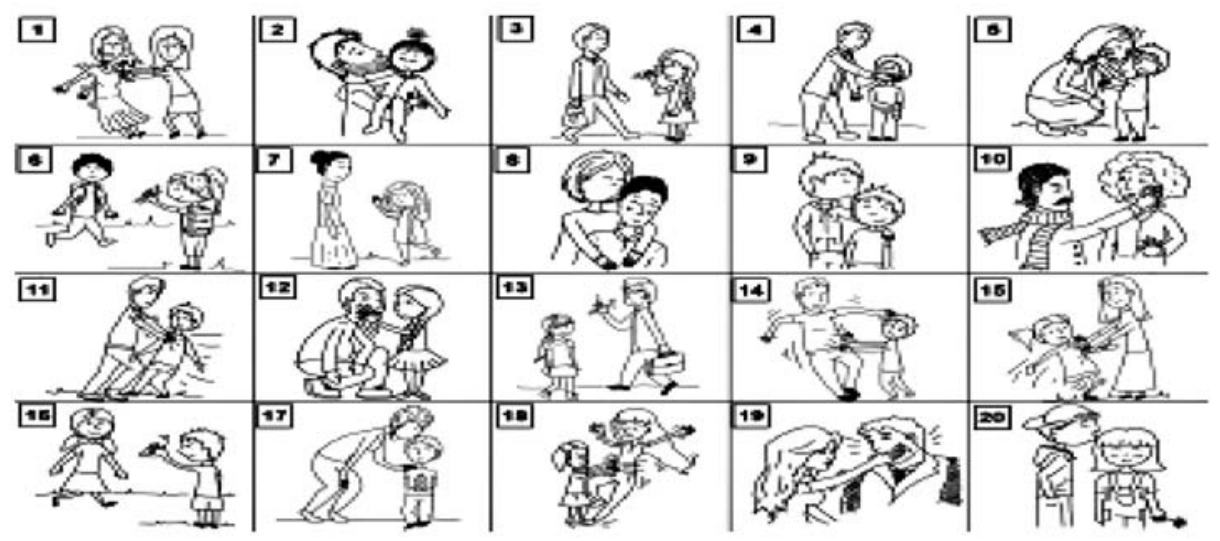

Fig. 1. Sample stimuls 

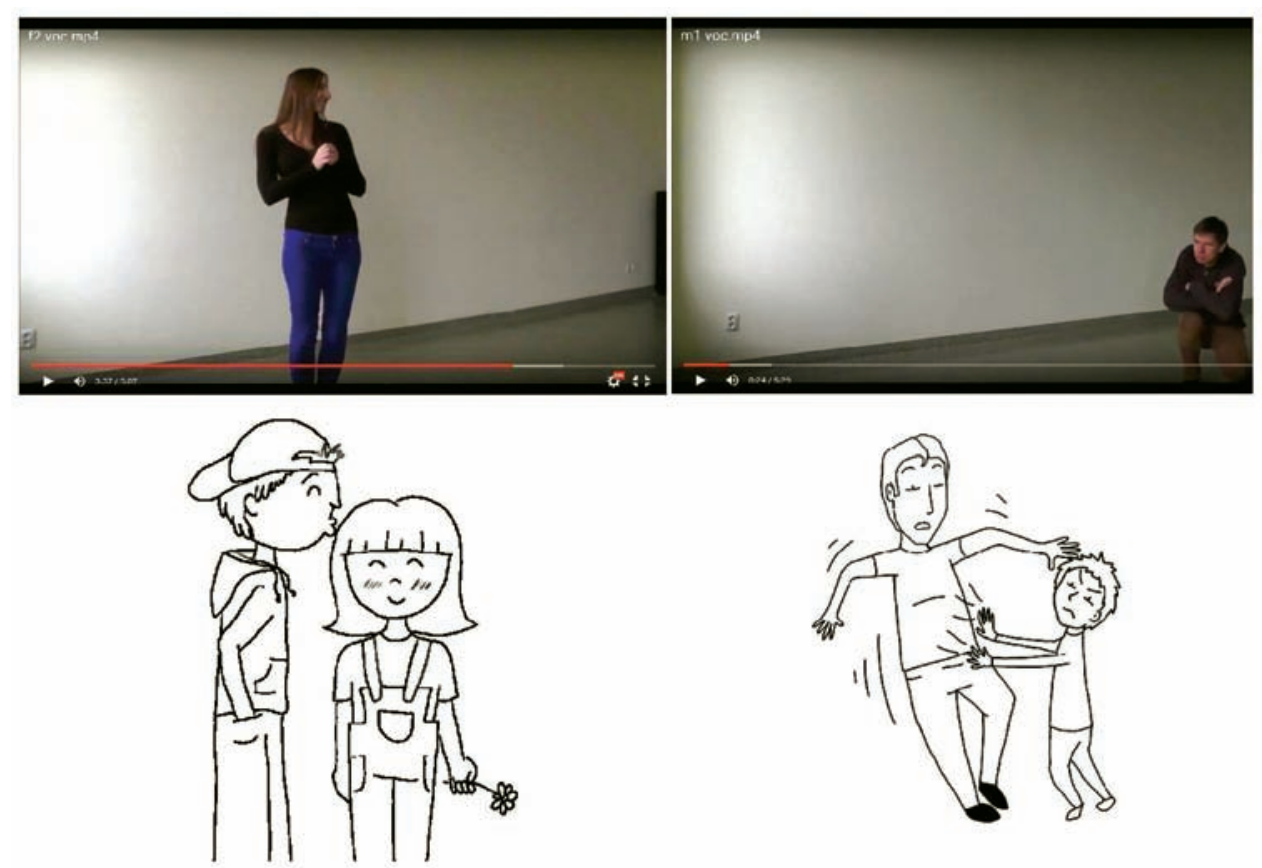

Fig. 2. Stills from the videos - characteristic features

common than SOV, which, according to the noisy channel hypothesis, should be opted against. Thus, the role-conflict constraint seems to be a more plausible factor influencing the sequencing of events. Although the initial results confirm the intuition proposed by Goldin-Meadow et al. that syntax of a spoken language may not necessarily influence the events reconstructed in gesture or by means of bodily representation, we cannot disregard the influence of the Polish syntax on the pantomimic re-enactments. This influence will be tested against more examples in our follow-up studies.

\section{References:}

Chomsky, N. (1957). Syntactic Structures. The Hague: Mouton.

Crystal, D. (2008). A Dictionary of Linguistics and Phonetics. Oxford: Blackwell.

Donald, M. (1991). Origins of the Modern Mind: Three Stages in the Evolution of Culture and Cognition. Cambridge, MA: Harvard University Press.

Dryer, M.S. (2005). The Order of Subject, Object and Verb. In: M. Haspelmath, M.S. Dryer, D. Gil, \& B. Comrie (Eds.), The World Atlas of Language Structures (pp. 330-333). Oxford: Oxford University Press. 
Gell-Mann, M., \& Ruhlen, M. (2011). The Origin and Evolution of Word Order, Proceedings of the National Academy of Sciences USA, 108(42), pp. 17290-17295.

Gershkoff-Stowe, L., \& Goldin-Meadow, S. (2002). Is There a Natural Order for Expressing Semantic Relations?, Cognitive Psychology, 45(3), pp. 375-412.

Gibson, E., Piantadosi, S.T., Brink, K., Bergen, L., Lim, E., \& Saxe, R. (2013). A Noisy-Channel Account of Crosslinguistic Word-Order Variation, Psychological Science, 24(7), pp. 1079-1088.

Goldin-Meadow, S., So, W., Özyürek, A., \& Mylander, C. (2008). The Natural Order of Events: How Speakers of Different Languages Represent Events Nonverbally, Proceedings of the National Academy of Sciences, 105(27), pp. 9163-9168.

Hall, M.L., Mayberry, R.I., \& Ferreira, V.S. (2013). Cognitive Constraints on Constituent Order: Evidence from Elicited Pantomime, Cognition, 129(1), pp. 1-17.

McNeill, D. (1992). Hand and Mind: What Gestures Reveal about Thought. Chicago: University of Chicago Press.

Meir, I., Lifshitz, A., Ilkbasaran, D., \& Padden, C.A. (2010, April). The interaction of animacy and word order in human languages: A study of strategies in a novel communication task. Paper presented at the 8th international conference on the evolution of language. Utrecht, Netherlands.

Pagel, M. (2009). Human Language as a Culturally Transmitted Replicator, Nature Reviews Genetics, 10(6), pp. 405-415.

Sandler, W., Meir, I., Padden, C., \& Aronoff, M. (2005). The Emergence of Grammar: Systematic Structure in a New Language, Proceedings of the National Academy of Sciences, 102(7), pp. 2661-2665.

Shannon, C. \& Weaver, W. (1949). The mathematical theory of communication. Urbana, IL: University of Illinois Press.

Zlatev, J. (2007). Embodiment, Language and Mimesis. In: T. Ziemke, J. Zlatev, \& R. Frank (Eds.), Body, Language and Mind (pp. 297-337). Berlin: Walter de Gruyter.

Internet sources:

Pre-print for The Encyclopaedia of Language and Linguistics, Gesture: A Psycholinguistic Approach, p. 3. Retrieved from: http://mcneilllab.uchicago.edu/pdfs/gesture.a_psycholinguistic_approach.cambridge.encyclop.pdf (DOA: 18.02.2016). 